\title{
EDITORIAL
}

\section{Apnea, intermittent hypoxia and blood transfusions: it works, but now what?}

Journal of Perinatology (2014) 34, 881; doi:10.1038/jp.2014.145

Apnea of prematurity and recurrent hypoxemia episodes occur very frequently in preterm infants, especially in those less than 28 weeks gestational age. Despite an extensive literature on the pathophysiology, epidemiology and clinical course of apnea of prematurity, it remains unclear whether it is harmful to the developing brain. Limited data suggest that the total number of days with apnea and later resolution of episodes are associated with worse neurodevelopmental outcome in premature infants. ${ }^{1}$ However, it is difficult to separate any potential adverse effects of apnea from the degree of immaturity at birth, as the incidence of apnea is inversely proportional to gestational age.

With more intensive monitoring and recording techniques including high-resolution oximetry with short averaging times and sampling rates, it is clear that clinically recognized apneic spells are the 'tip of the iceberg' in the symptomatology of immature respiratory control in premature infants. Intermittent hypoxia $(\mathrm{IH})$ episodes (defined as brief, repetitive cycles of decreases in oxygen saturation from baseline), which may be clinically unapparent with oximetry averaging times used in the clinical setting, occur frequently in premature infants. ${ }^{2} \mathrm{IH}$ episodes can be documented to occur more than 400 times a day in convalescent very low birth weight infants, and are also observed in infants considered to have clinically resolved apnea, as well as after discharge to home. ${ }^{3,4}$ The potential neurodevelopmental impact of these often severe hypoxia events in the preterm infant is unknown. In one study, a greater frequency of $\mathrm{IH}$ in extremely low birth weight infants was associated with worse severity of retinopathy of prematurity. ${ }^{3}$ In addition, in animal models and adults with sleep apnea $\mathrm{IH}$ is associated with a proinflammatory/prooxidant cascade, which could potentially cause injury to multiple organs including the brain. ${ }^{2}$ Studies to correlate the incidence and severity of $\mathrm{IH}$ events in preterm infants with adverse outcomes, and to examine whether more aggressive treatment is beneficial, remain a critical gap in our current knowledge.

Red blood transfusions have long been included as a potential treatment for apnea in anemic premature infants. However, results of studies to assess the impact of blood transfusions on recurrent apneic spells have shown mixed results. ${ }^{5-8}$ These studies have been hampered by small sample size, lack of masking of the intervention, differences in monitoring and recording techniques for apnea and/or desaturation, and reliance of nursing observations of apnea, which are known to be inaccurate. However, more recently with the use of continuous electronic recording of cardiorespiratory signals and oximetry, Zagol et al. ${ }^{9}$ showed that apnea episodes were less frequent in a cohort of very low birth weight infants for the 3 days after compared with 3 days before blood transfusion. In addition, apnea and desaturation episodes were found to be more common in gestational age matched preterm infants with lower hemoglobin levels compared to those with higher. ${ }^{9}$

In this edition of the Journal, Abu Jawdeh et al. ${ }^{10}$ add important new information to the association of anemia and apnea in preterm infants. High-resolution oximetry was continuously electronically recorded in a cohort of 130 infants born between
24 to 28 weeks gestation who received blood transfusions. They compared recorded $\mathrm{IH}$ events pre-transfusion with 24 and $48 \mathrm{~h}$ after transfusion over three postnatal age epochs ( 0 to 7 days, 8 days to 4 weeks and $>4$ weeks). The frequency and severity of $\mathrm{IH}$ episodes were significantly reduced up to $48 \mathrm{~h}$ after a RBC transfusion, but only in the infants $>1$ week of age. In addition, in the infants $>4$ weeks of age, the percent time spent with a saturation $<80 \%$ was also significantly decreased up to $48 \mathrm{~h}$ after the blood transfusion. However, the improvements, although statistically significant, were modest at best, with a decrease in the number of $\mathrm{IH}$ events in infants $>4$ weeks of age from a mean of approximately $400 /$ day to $300 /$ day, and an increase in the nadir of oxygen saturation from a mean of 72 to $74 \%$.

So, what does this mean clinically? We may finally have an answer to the question of blood transfusions and reduction in apnea and $\mathrm{IH}$ in convalescent premature infants-it works, but the duration of the benefit remains unclear as studies have not examined the effect beyond $72 \mathrm{~h}$. More importantly, does it matter? That conclusion awaits further understanding of the shortand long-term impact of $\mathrm{IH}$ on neurodevelopmental and other outcomes in preterm infants, and whether therapy for $\mathrm{IH}$ (with prolonged caffeine ${ }^{4}$ or maintenance of higher hemoglobin thresholds) affects those outcomes. Until we have more data, Abu Jawdeh's study suggests that judicious use of red cell transfusions in premature infants $>1$ week of age with severe apnea or $\mathrm{IH}$ events may be properly considered as short-term management.

EC Eichenwald

Department of Pediatrics, University of Texas Medical School, Houston, TX, USA

E-mail: eric.c.eichenwald@uth.tmc.edu

\section{REFERENCES}

1 Janvier A, Khairy M, Kokkotis A, Cormier C, Messmer D, Barrington KJ. Apnea is associated with neurodevelopmental impairment in very low birth weight infants. J Perinatol 2004; 24: 763-768.

2 Martin RJ, Wang K, Koroglu O, Di Fiore J, Kc P. Intermittent hypoxic episodes in preterm infants: do they matter? Neonatology 2011; 100: 303-310.

3 Di Fiore JM, Bloom JN, Orge F, Schutt A, Schluchter M, Cheruvu VK et al. A higher incidence of intermittent hypoxemic episodes is associated with severe retinopathy of prematurity. J Pediatr 2010; 157: 69-73.

4 Rhein LM, Dobson NR, Darnall RA, Corwin MJ, Heeren TC, Poets CF et al. Effects of caffeine on intermittent hypoxia in infants born prematurely: a randomized trial. JAMA Pediatr 2014; 168: 250-257.

5 Joshi A, Gerhardt T, Shandloff $P$, Bancalari E. Blood transfusion effect on the respiratory pattern of preterm infants. Pediatrics 1987; 80: 79-84.

6 DeMaio JG, Harris MC, Deuber C, Spitzer AR. Effect of blood transfusion on apnea frequency in growing premature infants. J Pediatr 1989; 114: 1039-1041.

7 Sasidharan P, Heimler R. Transfusion-induced changes in the breathing pattern of healthy preterm anemic infants. Pediatr Pulmonol 1992; 12: 170-173.

8 Stute $\mathrm{H}$, Greiner B, Linderkamp O. Effect of blood transfusion on cardiorespiratory abnormalities in preterm infants. Arch Dis Child Fetal Neonatal Ed 1995; 72: F194-F196.

9 Zagol K, Lake DE, Vergales B, Moorman ME, Paget-Brown A, Lee $\mathrm{H}$ et al. Anemia, apnea of prematurity, and blood transfusions. J Pediatr 2012; 161: 417-421, e411.

10 Abu Jawdeh EG, Martin RJ, Dick TE, Walsh MC, Di Fiore JM. The effect of red blood cell transfusion on intermittent hypoxemia in ELBW Infants. J Perinatol 2014; 34: 921-925. 